\title{
Infection Prevention and Control for Ebola in Health Care Settings - West Africa and United States
}

\author{
Jeffrey C. Hageman, MHS ${ }^{1}$ \\ Carmen Hazim, $\mathrm{MPH}^{1,2}$ \\ Katie Wilson, $\mathrm{MPH}^{1}$ \\ Paul Malpiedi, $\mathrm{MPH}^{1}$ \\ Neil Gupta, MD ${ }^{1}$ \\ Sarah Bennett, $\mathrm{MD}^{3}$ \\ Amy Kolwaite, $\mathrm{MPH}^{1}$ \\ Abbigail Tumpey, $\mathrm{MPH}^{1}$ \\ Kristin Brinsley-Rainisch, MPH ${ }^{1}$ \\ Bryan Christensen, $\mathrm{PhD}^{1}$ \\ Carolyn Gould, $\mathrm{MD}^{1}$ \\ Angela Fisher ${ }^{1}$ \\ Michael Jhung, MD ${ }^{4}$ \\ Douglas Hamilton, $\mathrm{MD}^{5}$ \\ Kerri Moran ${ }^{1}$ \\ Lisa Delaney, MS 6 \\ Chad Dowell, $\mathrm{MS}^{6}$ \\ Michael Bell, $\mathrm{MD}^{1}$ \\ Arjun Srinivasan, $\mathrm{MD}^{1}$ \\ Melissa Schaefer, $\mathrm{MD}^{1}$ \\ Ryan Fagan, $\mathrm{MD}^{1}$ \\ Nedghie Adrien ${ }^{2}$ \\ Nora Chea, $\mathrm{MD}^{1}$ \\ Benjamin J. Park, MD ${ }^{1}$ \\ ${ }^{1}$ Division of Healthcare Quality Promotion, National Center for Emerging and Zoonotic Infectious Diseases, CDC \\ ${ }^{2}$ Oak Ridge Institute for Science and Education, Oak Ridge, Tennessee \\ ${ }^{3}$ Division of Global Health Protection, Center for Global Health, CDC \\ ${ }^{4}$ Influenza Division, National Center for Immunization and Respiratory Diseases, CDC \\ ${ }^{5}$ Division of Global Migration and Quarantine, National Center for Emerging and Zoonotic Infectious Diseases, CDC \\ ${ }^{6}$ National Institute for Occupational Safety and Health. CDC
}

Corresponding author: Jeffrey C. Hageman, Division of Healthcare Quality Promotion, National Center for Emerging and Zoonotic Infectious Diseases, CDC. Telephone: 404-639-4951; E-mail: JHageman@cdc.gov.

\section{Summary}

The 2014-2016 Ebola virus disease (Ebola) epidemic in West Africa underscores the need for health care infection prevention and control (IPC) practices to be implemented properly and consistently to interrupt transmission of pathogens in health care settings to patients and health care workers. Training and assessing IPC practices in general health care facilities not designated as Ebola treatment units or centers became a priority for CDC as the number of Ebola virus transmissions among health care workers in West Africa began to affect the West African health care system and increasingly more persons became infected. CDC and partners developed policies, procedures, and training materials tailored to the affected countries. Safety training courses were also provided to U.S. health care workers intending to work with Ebola patients in West Africa. As the Ebola epidemic continued in West Africa, the possibility that patients with Ebola could be identified and treated in the United States became more realistic. In response, CDC, other federal components (e.g., Office of the Assistant Secretary for Preparedness and Response) and public health partners focused on health care worker training and preparedness for U.S. health care facilities. CDC used the input from these partners to develop guidelines on IPC for hospitalized patients with known or suspected Ebola, which was updated based on feedback from partners who provided care for Ebola patients in the United States. Strengthening and sustaining IPC helps health care systems be better prepared to prevent and respond to current and future infectious disease threats.

The activities summarized in this report would not have been possible without collaboration with many U.S. and international partners (http://www.cdc.gov/vhflebolaloutbreaks/2014-west-africalpartners.html). 


\section{Background}

Infection prevention and control (IPC) is an essential, ongoing requirement to protect patients and health care workers (HCWs) from the spread of infectious diseases in health care settings. The 2014-2016 Ebola virus disease (Ebola) epidemic in West Africa underscored how actions in health care settings can contain or amplify an infectious disease threat in a community. Failure to effectively and consistently implement IPC practices can lead to outbreaks among HCWs and patients. A smaller workforce after an outbreak might require the closing of facilities, as occurred during the 2014-2016 Ebola epidemic. Closing of a facility not only affects outbreak control but can jeopardize the delivery of care (e.g., routine, trauma, cardiac emergency, and obstetric care).

During outbreaks of new and emerging infectious diseases, CDC provides guidance on IPC for U.S. health care settings to prevent spread of infections within health care facilities; coordinates and engages experts throughout CDC and federal and state partners for activities, such as developing additional guidance or tools; and communicates with and engages stakeholders to reach the broadest audience. During the Ebola epidemic, CDC expanded its domestic role and provided guidance and leadership internationally to train and educate HCWs in affected countries in West Africa and in the United States.

\section{CDC Contributions and Impact}

\section{International Infection Prevention and Control}

Early in the Ebola epidemic, Ebola transmission to HCWs occurred in health care facilities that were not Ebola treatment units (ETUs) (1-3). Health care facility assessments conducted by CDC and partners in 2014 documented substantial gaps in IPC. These gaps (i.e., a lack of IPC oversight, poor waste management procedures, a lack of triage and isolation protocols, frequent lack or misuse of personal protective equipment $[\mathrm{PPE}]$, and inadequate standard infection control precautions) increased the risk for Ebola transmission in nonETU health care settings $(4,5)$.

Beginning in August 2014, CDC developed partnerships with ministries of health, the World Health Organization (WHO), and others to improve IPC rapidly at non-ETU health care facilities and to decrease the risk for Ebola transmission to HCWs. A critical first step was to establish national IPC task forces to coordinate infection control efforts within Guinea, Sierra Leone, and Liberia. Before these task forces were established, numerous organizations working to improve IPC within the affected countries had developed training materials that sometimes gave conflicting technical details and led to confusion among HCWs. The establishment of ministry of health-supported national IPC task forces within each country improved communication among partners and coordinated the development of technically sound and consistent standard operating procedures relevant for resource-limited clinical settings. These standard operating procedures listed procurement of PPE and other IPC supplies for health care facilities.

Along with the establishment of national IPC task forces, CDC and partners developed and trained local and facilitylevel IPC leadership, also called IPC specialists or focal persons. IPC specialists oversaw IPC at facilities and led ongoing facility IPC improvements, including providing HCW training and ensuring availability of supplies. In addition to conducting IPC training, CDC and partners provided onsite mentorship and supportive supervision to rapidly implement IPC improvements. In Liberia, the first cadre of IPC specialists included medical residents and physicians from hospitals that closed because of the epidemic. These specialists supported 10-15 hospitals, health centers, and clinics in 14 of 15 counties in the country. In Guinea, IPC specialists, trained and funded by partner organizations, were overseeing triage and IPC at large hospitals in the short term. In Sierra Leone, the Ministry of Health and Sanitation appointed permanent IPC specialists for the 25 Government hospitals in February 2015.

In addition to helping establish IPC policies and procedures, CDC also worked with partners to develop standard IPC training materials specific for available resource levels that were then tailored (e.g., translated into different languages) for use in the affected countries. These outlined the IPC practices that needed to be implemented in health care facilities, community care centers, patient transport systems, and communities (G). After CDC technical review of materials, IPC partners launched efforts intended to train HCWs in each of the three countries on proper screening, isolation, and notification procedures for patients arriving at non-ETU facilities. CDC staff participated in the trainings using a train-the-trainers framework, resulting in at least 765 master trainers delivering training to approximately 24,000 HCWs in Liberia, Guinea, and Sierra Leone.

To supplement efforts to strengthen IPC practices systemwide, a new strategy known as Ring IPC was introduced in which rapid, intensive, and short-term IPC support is delivered to health care facilities in areas of active Ebola transmission to help break the chain of transmission ( 7 ). Once high-risk facilities were identified, IPC assessments were conducted to guide technical assistance, medical supply distribution, and daily supportive supervision to ensure HCWs were trained to triage, isolate, and refer suspected and probable Ebola patients rapidly to ETUs. 
Ring IPC impacted several places. For example, in Liberia, three febrile HCWs were identified when screened for work; all were properly isolated and transferred to an ETU for testing (7). Sierra Leone integrated Ring IPC around clusters of Ebola patients in three districts. Guinea focused on minimizing transmission by rapidly investigating infected HCWs and remediating IPC lapses.

\section{Training U.S. Health Care Workers Traveling to West Africa}

The large number of infected HCWs caused workforce shortages in the three countries that were most heavily affected. Clinical staff from countries around the world, including the United States, volunteered to care for Ebola patients in ETUs. Although training courses, such as those offered by Médecins Sans Frontières (MSF) and WHO, had been developed to prepare ETU workers, requests for enrollment in available training courses exceeded capacity. In addition, no similar courses in the United States met the need for training U.S. clinicians on providing safe care for patients in West Africa.

To address the safety of U.S. medical volunteers, CDC formed a task force that developed a 3-day safety training course for U.S. HCWs intending to work in West Africa ETUs (8). Task force members traveled to Belgium in August 2014 to participate in the MSF course. With the full collaboration and participation of MSF and WHO, the team used the two organizations' Ebola materials as the foundation for the CDC course curriculum.

CDC conducted the course, called Preparing Healthcare Workers to Work in Ebola Treatment Units (ETUs) in Africa, at the U.S. Federal Emergency Management Agency's Center for Domestic Preparedness in Anniston, Alabama. The team trained approximately $600 \mathrm{HCW}$ representing 42 nongovernment organizations and 21 institutions, organizations, and agencies of the U.S. government. Of the HCWs trained, 276 were Commissioned Officers of the U.S. Public Health Service who staffed the Monrovia Medical Unit in Liberia during 2014-2015 (9) and provided care to infected HCWs. The training team also produced a tool kit of the training curriculum so that other organizations could replicate the course (10).

\section{Developing Ebola IPC Guidance for U.S. Health Care Facilities}

CDC is the lead federal agency for developing infection control guidance that U.S. health care facilities can use when implementing local protocols and procedures. This guidance is based on evidence found in published literature or gained from field experience. In situations where intervention is required for new or emerging infections and there is a paucity of data available, CDC develops guidance based on the best information available (e.g., existing CDC guidance for similar diseases, current epidemiologic and laboratory information, peer-reviewed evidence, and expert opinion). These documents typically are written to provide flexibility in implementation to account for differences in facility-specific characteristics (e.g., facility design and types of supplies available) across health care settings (e.g., hospitals compared with outpatient settings).

In August 2014, anticipating the possibility that Ebola could be diagnosed and treated in the United States and knowing that no U.S. health care facility had experience treating Ebola, CDC infection control, occupational safety and health, and Ebola experts developed and disseminated Infection Prevention and Control Recommendations for Hospitalized Patients with Known or Suspected EVD in U.S. Hospitals (11). These recommendations included guidance on patient placement, PPE use, aerosol-generating procedures, environmental infection control, monitoring and management of potentially exposed HCWs, and other critical aspects of prevention of Ebola transmission in hospitals. At the time, the recommended PPE for Ebola patient care was a gown, gloves, eye protection, and facemask; additional PPE (e.g., shoe covers, leg covers, double gloving, and respirator) for HCWs was recommended if the HCW anticipated contact with copious body fluids or would be performing aerosol-generating procedures. In September 2014, CDC and the Office of the Assistant Secretary for Preparedness and Response (ASPR) advised that all hospitals should prepare for the possibility that persons in West Africa with Ebola could travel to the United States and distributed a checklist to guide hospitals' preparedness (12).

The importation of an Ebola case to a Dallas, Texas, health care facility and the subsequent spread of Ebola to two nurses who provided care demonstrated that HCW PPE recommendations needed to be more directive (e.g., only two recommended PPE ensemble options) and be standardized to facilitate training efforts as well as to ensure the proper supply distribution of the recommended PPE. In addition, CDC received feedback from partners, including those who had provided care for the Ebola patients in the United States, regarding invasive procedures or changes to routine processes (e.g., patient care staff remained in patient's room for extended periods). As a result of these experiences, CDC updated the Ebola PPE guidance for HCWs in October 2014, emphasizing that facilities should choose a single standardized PPE approach for patient care, provide training and document competency in PPE use, and use a trained observer during donning and doffing. The observer would help to ensure that PPE was donned correctly and would alert the HCW to 
possible contact with body fluids during doffing of used PPE (13). CDC received input from other federal agencies with regulatory oversight of health care and occupational safety and health issues, including the Food and Drug Administration, the Occupational Safety and Health Administration, and the National Institutes of Health. Feedback was also received from nongovernment professional medical societies and organizations and public health authorities with expertise in Ebola, IPC, and occupational safety and health. Other sources of feedback were hospital staff who had safely cared for Ebola patients in the United States (Emory University Hospital in Atlanta, Georgia, and Nebraska Medical Center in Omaha, Nebraska) and in Africa (MSF).

\section{Training and Educating HCWs in U.S. Health Care Facilities}

Updating the infection control guidance was an important step to provide additional specificity; however, the delivery of information and the requirements for implementation needed to be strengthened. Challenges included the differing levels of preparedness among U.S. health care facilities, variations in HCW' roles and their baseline levels of infection control knowledge and training, and differences in the amount and types of infection control supplies (e.g., PPE) available to HCWs. To address these challenges, CDC developed partnerships with a diverse group of organizations to develop educational resources applicable to various settings and $\mathrm{HCW}$ types. CDC deployed teams to assess infection control readiness at facilities being designated by state authorities to care for and assess Ebola patients, with the goal of creating training and educational resources based on CDC guidance that are action-oriented, modular, accessible on mobile devices for on-demand use, available in multiple formats, and endorsed by key stakeholders. These tools also took into account best practices related to adult learning, risk communication, and clear communication. CDC training was delivered by using a multifaceted approach: onsite technical assistance, Web-based tools, video training and resources, webinars and conference calls, and in-person training.

\section{Onsite Technical Assistance}

CDC and ASPR collaborated with state health departments to improve facility readiness by assessing facilities that can safely care for a patient with Ebola and develop guidance to prepare U.S. health care facilities for Ebola. Facilities were designated in three tiers (14): Ebola treatment centers (ETCs) $(14,15)$, assessment hospitals $(14,16)$, and frontline health care facilities $(14,17)$. Fifty-five state-designated ETCs were designated by state health authorities by February 2015, of which nine serve as regional treatment centers. ETCs are staffed, equipped, and have been assessed for their ability to provide care for an Ebola patient for the complete duration of illness. CDC teams assessed infection control readiness by visiting 81 facilities in 21 states and the District of Columbia that were being considered to serve as ETCs by January 2015.

CDC and ASPR worked with state and local public health officials to identify Ebola assessment hospitals through Ebola readiness assessment teams. These hospitals are intended to have the capability to evaluate and care for persons suspected of having Ebola for up to 96 hours, initiate or coordinate Ebola testing, and test for alternative illnesses. These hospitals can transfer patients to an ETC as needed. Ebola readiness assessment teams assess facilities for key capacities, including staff training, infection control, and PPE use. Through December 2015, Ebola readiness assessment teams assessed approximately 40 facilities.

\section{Web-Based Tools}

CDC's Ebola HCW Web pages, which feature training videos and materials (e.g., job aids such as algorithms and checklists), were successively tailored during the fall of 2014 to accommodate the growing needs of the response (18). Usability testing was conducted with stakeholders before the third redesign in December 2014 to ensure that users could easily access CDC's guidelines and training information. The Ebola HCW Web pages were viewed approximately 7.5 million times in fall of 2014.

\section{Web-Based Video Training}

Effectively donning and doffing PPE are two of the most complex actions for HCWs caring for an Ebola patient. On October 31, 2014, within 11 days after releasing updated PPE guidance, CDC and Johns Hopkins Armstrong Institute for Patient Safety used human factors engineering methods to develop and launch an interactive Web-based video learning program detailing procedures for four main PPE combinations (19). To support facility preparedness, four Web-based video training modules were included for emergency department personnel in ETCs and Ebola assessment hospitals, providing detailed instructions on safely assessing and caring for patients with Ebola and other infectious diseases (20). By July 31, 2015, the PPE video modules had been viewed 576,410 times, with an estimated 518,682 minutes $(8,644$ hours) watched. The emergency department training modules were viewed a total of 15,675 times, with an estimated 1,405 hours watched.

\section{Webinars and Conference Calls}

CDC conducted approximately 160 webinars and conference calls, reaching approximately 160,000 U.S. health 
care providers. Most of these calls were conducted during July 2014-January 2015 in collaboration with clinical professional partners (e.g., American Hospital Association).

\section{Resources on Clinician-Specific Websites}

During the fall of 2014, online clinical communities (e.g., Medscape) provided substantial additional outreach to U.S. health care providers. The public-private partnership between $\mathrm{CDC}$ and WebMD/Medscape enables rapid dissemination of urgent training and information to clinicians during public health crises. Medscape produced eight video expert commentaries and a short how-to video on donning and doffing PPE when caring for Ebola patients and collaborated with CDC to address questions from health care professionals (21). The Ebola commentaries on Medscape were viewed approximately 386,000 times and have been promoted and used for HCW training throughout the United States and internationally.

\section{In-Person Training}

CDC linked with Partnership for Quality Care and numerous health care organizations and unions to conduct live training events in New York, New York; Los Angeles, California; and Philadelphia, Pennsylvania; these events reached approximately 6,500 individuals in person and approximately 20,000 through live webcast $(22,23)$. In addition, CDC, Emory University Hospital, and Nebraska Medical Center trained approximately 1,000 HCWs from designated ETCs, Ebola assessment hospitals, and state health departments on all aspects of infection control and patient care for Ebola patients. On July 1, 2015, CDC and ASPR announced the launch of the National Ebola Training and Education Center, led by three institutions (Emory University Hospital, Nebraska Medical Center, and Bellevue Hospital in New York, New York) to continue and expand on efforts to ensure health care facilities and biocontainment centers maintain readiness to care for patients with Ebola in the United States (24).

\section{Conclusion}

Even after Ebola cases in West Africa have declined to zero, the infection control safety net must be sustained to prevent reemergence of the epidemic, and the lessons learned from this response augmented to improve infection control in U.S. health care facilities and globally. Emerging infectious diseases such as Ebola will inevitably occur, possibly without warning. Hospitals and other health care facilities must remain vigilant and prepared to implement prompt triage of potentially infectious patients and maintain recommended infection control practices during all patient care activities, regardless of patients' known infection status.
As of March 2016, a total of 261 CDC or other U.S. government staff had deployed to West Africa to support IPC efforts. These IPC efforts have resulted in numerous improvements in safety and most likely have prevented infection in many patients and HCWs. Triage procedures were established at nearly all non-ETU key health care facilities, with trained staff to screen for suspected cases at entry points. HCWs, now trained on the use of PPE for standard and Ebola-specific precautions, routinely provide care using appropriate PPE. IPC specialists in Guinea, Sierra Leone, and Liberia have overseen numerous IPC improvements in waste management, hand hygiene, environmental decontamination, and other critical facility safety components. One of the last cases in Liberia, a symptomatic person with no known contact with an Ebola patient, was identified by a triage nurse before entry to the hospital; subsequent isolation resulted in no health care-associated cases or exposures (25). Overall, the number of HCW infections has declined dramatically, as has the proportion of cases occurring among HCWs (1). Taken together, IPC efforts have greatly reduced the likelihood of transmission in a health care setting, one of the major settings for Ebola transmission during this epidemic (1).

In West Africa, strengthening and sustaining IPC in health care systems established for the epidemic will help prevent future disease transmission. Equipment, supplies, and infrastructure are all essential elements of IPC, and access to them will need to be ensured. International partners will need to ensure that, at a minimum, HCWs always have access to gloves, especially at primary care points, such as hospitals, clinics, and other facilities where the risk for transmission is high. Reliable water, electricity, and waste disposal at health care facilities are critical, and such infrastructure improvements would further contribute to decreasing disease transmission in West Africa. In addition, effective, sustainable, and scalable lower-cost solutions, such as local production of alcohol-based hand rub, are needed.

In the United States, sustaining the education, training, and competency of HCWs on IPC practices is needed not only to prepare for emerging threats but also to prevent transmission of endemic disease in U.S. health care facilities. Common health-care-associated infections alone are responsible for substantial numbers of illnesses and even deaths among patients; in 2011, an estimated 722,000 U.S. patients acquired health-care-associated infections, and 75,000 died (26). Emphasis on microbiology and IPC principles for all $\mathrm{HCW}$ s that begins during medical, nursing, and other clinical education programs can help provide a foundation for safe care.

The Ebola response also highlighted the need for research and evaluation of new infection control practices and technologies to ensure that the safety of care keeps pace with 
the ever-evolving health care system. Opportunities for health care innovations include ways to:

- improve the detection, triage, and isolation process for potentially infectious patients seeking care; ensure HCW and patient safety; and prevent spread within health care systems;

- upgrade the design and construction of health care facilities to make infection control as effective as possible (e.g., by eliminating crowded waiting rooms and providing pathways for patient triage and transport of infectious material);

- adopt innovative technologies for environmental cleaning and disinfection; and

- improve the design of PPE for health care so that it meets the needs of the personnel caring for patients (e.g., PPE designed to allow effective patient care interactions and facilitate removal without risk of contaminating the environment or the wearer).

In addition to training HCWs, implementing IPC across health care systems requires improving accountability and incentives to support sustained change, providing evidencebased interventions and solutions to support facility improvements, and using public health data to track progress. As these processes are implemented, health care systems will be better prepared to prevent and respond to current and future infectious disease threats.

\section{Acknowledgments}

International acknowledgments: Mary Hamel, Jennifer MacFarquhar, Alexis Harvey, Rachel Smith, Alyssa Finley, David Fitter, David Miller, Elise Rodd, Emma Prasher, Eric Mintz, Fatima Ravat, Heidi Soeters, Hoang Dang, Lindsey Horton, Monica Patton, N’Deye Rosalie Niang, Ian Plumb, Negar Aliabadi, Siré Camara, Ellen Dotson, Evan Shukan, Bi Kakou Djassa, G. Laissa Ouedraogo, Melissa Briggs-Hagen, Jevon McFadden, Nadezhda Duffy, Megan Klingler, Kevin Clarke, Lauren Lewis, Jenny Lin, Sandra Jackson, Snigdha Vallabhaneni, Elinor Greene, Ben Levy, Carrie Nielsen, Leah Gilbert, Kendra Broadwater, Ibrahim Kamara, Eddie Jackson, Sophia Greer, Rey de Castro, Christopher Fletcher, Fatma Soud, Gabe McLemore, Alison Ridpath, Brooke Hoots, Eugene Lam, Ethel Taylor, Eric Esswein, Laura Cooley, Chris Gregory, Brenda Baker, Kipruto Chesang.

Domestic acknowledgements: Steve Boedigheimer, Mark Davis, Todd Talbert, Sherline Lee, Dahna Batts, and others on the State Coordination Task Force; John Brooks, Denise Jameson, Cynthia Whitney, Dana Meaney Delman, Peter Briss, Sue Gerber, John Jernigan, Joe Perz, and others on the Medical Care Task Force and Infection Control teams; Barbara Mahon, Paul Mead, Barbara Knust, Stephanie Bialek, Alicia Fry, Monina Klevens, Margaret (Peggy) Honein, Lyn Finelli, Gayle Langley, and others on the Epidemiology Team; Elissa Meites, Emily Koumans, Roxanne Barrow, Mary Kamb, LeAnne Fox, and others assisting with Domestic Inquiries; Stephanie Scrag, Alex Kallen, Chris Braden, Todd Weber, and the many persons who worked on the ground in Texas, Ohio, and New
York City; Nicki Peski, Nicole Cohen, Clive Brown, and others on the Global Migration Task Force; Eric Kasowski, Inger Damon, and Jordan Tappero; Chief of Staff staff, including Sherrie Bruce, David Hutchins, and Stephen Papagiotas; and Anita Patel, Jeff Nemhauser, and Lauri Hicks, for leading efforts to assist CDC staff returning from West Africa; John Decker, Maryann D’Allesandro, Ron Shaffer, Selcen Kilinc-Balci, Christina Williams, Nicole Coffin, Rosa Herrera, Fred Maxineau, Cynthia Baur, John Horan, Peter Costa, Jennie Johnston, Adam Arthur, Nadia Ennis, Alfred Junior, Amy Nadel, Annic Jobin, Teresa Horan, Ayse Gurses, Lisa Maragakis, Glenn Platt, Neal Sikka, Jeff Wages, John O’Connor, Mary Choi, Dana Pitts, Rachel Kossover, Lacey Avery, Rachel Powell, Phyllis Silver.

\section{References}

1. World Health Organization. Health worker Ebola infections in Guinea, Liberia and Sierra Leone: preliminary report. Geneva, Switzerland: World Health Organization; 2015. http://www.who.int/csr/resources/ publications/ebola/health-worker-infections/en

2. Matanock A, Arwady MA, Ayscue P, et al. Ebola virus disease cases among health care workers not working in Ebola treatment units-Liberia, JuneAugust, 2014. MMWR Morb Mortal Wkly Rep 2014;63:1077-81.

3. Kilmarx PH, Clarke KR, Dietz PM, et al. Ebola virus disease in health care workers-Sierra Leone, 2014. MMWR Morb Mortal Wkly Rep 2014;63:1168-71.

4. Pathmanathan I, O'Connor KA, Adams ML, et al. Rapid assessment of Ebola infection prevention and control needs - six districts, Sierra Leone, October 2014. MMWR Morb Mortal Wkly Rep 2014;63:1172-4.

5. Forrester JD, Pillai SK, Beer KD, et al. Assessment of Ebola virus disease, health care infrastructure, and preparedness - four counties, southeastern Liberia, August 2014. MMWR Morb Mortal Wkly Rep 2014;63:891-3.

6. CDC. Non-U.S. healthcare settings. International infection control for healthcare workers. Atlanta, GA: US Department of Health and Human Services, CDC; 2016. http://www.cdc.gov/vhf/ebola/hcp/non-us-healthcare-settings.html

7. Nyenswah T, Massaquoi M, Gbanya MZ, et al. Initiation of a ring approach to infection prevention and control at non-Ebola health care facilities_Liberia, January-February 2015. MMWR Morb Mortal Wkly Rep 2015;64:505-8.

8. CDC. Preparing healthcare workers to work in Ebola treatment units (ETUs) in Africa. Atlanta, GA: US Department of Health and Human Services, CDC; 2016. http://www.cdc.gov/vhf/ebola/hcp/safety-training-course/

9. Liberia: Monrovia Medical Unit Decommissioned. AllAfrica. 2016. http://allafrica.com/stories/201505051068.html

10. CDC. Preparing healthcare workers to work in Ebola treatment units (ETUs) in Africa: training toolkit announcement. Atlanta, GA: US Department of Health and Human Services, CDC; 2015. http://www. cdc.gov/vhf/ebola/hcp/safety-training-course/training-toolkit.html

11. CDC. Infection prevention and control recommendations for hospitalized patients under investigation (PUIs) for Ebola virus disease (EVD) in U.S. hospitals. Atlanta, GA: US Department of Health and Human Services, CDC; 2016. http://www.cdc.gov/vhf/ebola/healthcareus/hospitals/infection-control.html

12. Bedard P. CDC issues Ebola checklist: "Now is the time to prepare." Washington Examiner. September 15, 2014. http://m.washingtonexaminer. com/cdc-issues-ebola-checklist-now-is-the-time-to-prepare/article/2553396

13. CDC. Guidance on personal protective equipment to be used by healthcare workers during management of patients with Ebola virus disease in U.S. hospitals, including procedures for putting on (donning) and removing (doffing). Atlanta, GA: US Department of Health and Human Services, CDC; 2014. http://www.cdc.gov/vhf/ebola/hcp/procedures-for-ppe.html 
14. Van Beneden CA, Pietz H, Kirkcaldy RD, et al. Early identification and prevention of the spread of Ebola-United States. In: CDC's response to the 2014-2016 Ebola epidemic-West Africa and United States. MMWR Suppl 2016;65(Suppl No. 3).

15. CDC. Hospital preparedness: a tiered approach. Atlanta, GA: US Department of Health and Human Services, CDC; 2015. http://www. cdc.gov/vhf/ebola/healthcare-us/preparing/treatment-centers.html

16. CDC. Interim guidance for preparing Ebola assessment hospitals Atlanta, GA: US Department of Health and Human Services, CDC; 2015. http:// www.cdc.gov/vhf/ebola/healthcare-us/preparing/assessment-hospitals.html

17. CDC. Interim guidance for preparing frontline healthcare facilities for patients under investigation (PUIs) for Ebola virus disease (EVD). Atlanta, GA: US Department of Health and Human Services, CDC; 2015. http:/www.cdc.gov/vhf/ebola/healthcare-us/preparing/frontlinehealthcare-facilities.html

18. CDC. U.S. healthcare workers and settings. Atlanta, GA: US Department of Health and Human Services, CDC; 2014. http://www.cdc.gov/vhf/ ebola/healthcare-us/index.html

19. CDC. Guidance for donning and doffing personal protective equipment (PPE) during management of patients with Ebola virus disease in U.S. hospitals. Atlanta, GA: US Department of Health and Human Services, CDC; 2014. http://www.cdc.gov/vhf/ebola/hcp/ppe-training/index.html
20. CDC. Ebola preparedness: emergency department training modules. Atlanta, GA: US Department of Health and Human Services, CDC; 2015. http:/www.cdc.gov/vhf/ebola/healthcare-us/emergency-services/ emergency-department-training.html

21. Medscape. Medscape Ebola resource center. 2014. http://www.medscape. $\mathrm{com} /$ resource/ebola

22. Greater New York Hospital Association. Ebola training. 2014. http:// www.gnyha.org/ebolatraining

23. Partnership for Quality Care. Ebola educational session-California. 2014. http://pqc-usa.org/caeventebola/

24. US Department of Health and Human Services. HHS launches National Ebola Training and Education Center. Washington, DC: US Department of Health and Human Services; 2015. http://www.hhs.gov/news/ press/2015pres/07/20150701a.html

25. Christie A, Davies-Wayne GJ, Cordier-Lassalle T, et al. Possible sexual transmission of Ebola virus-Liberia, 2015. MMWR Morb Mortal Wkly Rep 2015;64:479-81.

26. Magill SS, Edwards JR, Bamberg W, et al. Multistate point-prevalence survey of health care-associated infections. N Engl J Med 2014;370:1198-208. 